\title{
Optical magnetic near-field intensities around nanometer-scale surface structures
}

\author{
Christian Girard \\ Laboratoire de Physique Moléculaire, Université de Franche-Comté, F-25030 Besançon, France \\ Jean-Claude Weeber and Alain Dereux \\ Laboratoire de Physique, Optique Submicronique, Université de Bourgogne, F-21011 Dijon, France \\ Olivier J. F. Martin \\ Swiss Federal Institute of Technology, ETH-Zentrum, CH-8092 Zurich, Switzerland \\ Jean-Pierre Goudonnet \\ Laboratoire de Physique, Optique Submicronique, Université de Bourgogne, F-21011 Dijon, France
}

(Received 18 February 1997)

\begin{abstract}
Recently, local probes used in optical experiments added a new dimension to the study of the optical properties of small particles lying on a surface. Until now, several theoretical frameworks, developed to understand the interaction of optical fields with mesoscopic and nanoscopic objects, emphasized mainly the prediction of the electric near-field distributions generated by these structures. This paper demonstrates how such subwavelength dielectric surface structures also produce a particular confinement of the optical magnetic near field when the sample is illuminated by a surface wave. [S0163-1829(97)06824-0]
\end{abstract}

\section{INTRODUCTION}

Current progress in nanofabrication techniques enables one to build well-defined low-symmetry surface structures. Since the first development of the near-field optics (NFO) instrumentation, the imaging of such small material particles lying on a surface or occurring in the vicinity of nanometersize structures became an active research area. ${ }^{1-4}$ Henceforth, the NFO local probe techniques offer a vast array of interesting opportunities, i.e., detecting evanescent fields in guiding structures, ${ }^{5,6}$ imaging and exciting localized plasmons over a metallic surface, ${ }^{7,8}$ mapping the structure of the optical electric field inside two-dimensional resonators tuned by adjustable mirrors, ${ }^{9}$ and performing subwavelength nearfield optical holography. ${ }^{10}$

In the past three years, different self-consistent studies indicated unambiguously that the individual structures lying on the surface distort the optical electric near-field intensity established by the self-consistent interaction between the surface roughness and the incident light. ${ }^{11-14}$ It was demonstrated that when the lateral dimensions of tiny objects are significantly smaller than the incident wavelength, the interference pattern collapses and the optical electric near-field intensity distribution tends to be fairly well localized around the objects. ${ }^{15-17}$ Under well-defined conditions on the incident field (polarization, wavelength) a highly localized electric near-field intensity occurs just above the subwavelength protrusions. In fact, when we deal with subwavelength objects, the importance of retardation effects decreases dramatically, so that the symmetry of the field distribution is governed only by the polarization of the incident field and the profile of the object itself. Recently this simple picture facilitated the interpretation of this peculiar NFO phenomenon. For example, a simple dielectric cube of cross section $100 \times 100 \mathrm{~nm}^{2}$ was imaged with the dielectric tip of a photon scanning tunneling microscope (PSTM) with a bright contrast when the surface wave was $p$ polarized and with a dark contrast when it was $s$ polarized. ${ }^{18}$ In this particular case, where the NFO image was recorded using a purely dielectric detector, precise theoretical modelings established a direct relation between the NFO image and the electric near-field intensity map computed a few nanometers away from the sample. ${ }^{16,17}$

On the other hand, when the detector extremity is either completely or partially covered with a thin metallic coating, both theoretical modelings and experimental measurements supply NFO images that do not follow the optical electric intensity anymore. ${ }^{19,20,14}$ The contrast appears to be significantly modified by the presence of the metallic coating and the information contained in the images must be reexamined by considering other optical effects. These results raise once again a fundamental problem in NFO, namely, the precise understanding of the tip-sample coupling in the near-field zone. Some time ago, Barchiesi and Van Labeke pointed out this serious problem using a reciprocal space perturbative method specially developed for NFO computations. ${ }^{21}$ In this paper, the possible role played by the optical magnetic field was brought to the fore. More recently, the consequences of metal coatings deposited on NFO probes was also discussed by Courjon et $a l^{22}$

In order to provide more insight into this complicated problem and thereby to get a better control of the imaging process in NFO, three important questions should be considered. (1) what kind of optical magnetic near-field maps can we expect around dielectric surface structures of subwavelength sizes? (2) How can one explain these nanometer-scale optical magnetic near fields? (3) How is it possible to detect them?

The main purpose of this paper is to address the two first questions theoretically. By using the same three-dimensional 
test objects that were used in Refs. 16, 18, and 20 we will compare the optical magnetic near-field intensities issued from two accurate Maxwell's equation solvers: (a) the direct space integral equation method ${ }^{16,17,23}$ (DSIEM) and (b) the differential theory of gratings (DTG). ${ }^{24-28}$

This paper is organized as follows: In Sec. II, we present a brief overview of the field susceptibility or Green's dyadic technique, which allows us to obtain general solutions of both electric and magnetic fields through two different vector Lippmann-Schwinger equations. In Sec. III, these integral equations will be solved by using the DSIEM already discussed in the NFO literature. ${ }^{23}$ In particular two generalized field propagators, independent of the illumination mode, will be introduced and applied in Sec. IV to systems of experimental interest. Finally, we shall conclude our study by comparing the DSIEM numerical results with those issued from the DTG Maxwell's equation solver.

\section{INTEGRAL EQUATION FORMALISM}

In this section, we present a brief overview of the concepts of both electric and magnetic field susceptibilities in the presence of a three-dimensional (3D) system of arbitrary shape and size. Starting from the microscopic Maxwell's equations expressed in terms of both charge and current densities, we express the response of a localized physical system submitted to an external electromagnetic excitation.

\section{A. Basic equations}

We begin now our detailed study of these optical fields by considering a spatially localized dielectric system of arbitrary shape characterized by its charge density $\rho(\mathbf{r}, t)$ and its current density $\mathbf{j}(\mathbf{r}, t){ }^{29,30,23}$ In NFO experimental circumstances, we can assume a monochromatic field with a time dependence of the form $e^{-i \omega t}$. Anyway, because Maxwell's equations are linear equations, the response of a given system to an arbitrary wave packet can be obtained from the superposition of the responses of this system to the individual plane waves forming the original wave packet. With this assumption, Maxwell's equations read in cgs units

$$
\begin{gathered}
\nabla \wedge \mathbf{E}(\mathbf{r}, \omega)=\frac{i \omega}{c} \mathbf{B}(\mathbf{r}, \omega), \\
\nabla \cdot \mathbf{B}(\mathbf{r}, \omega)=0, \\
\nabla \cdot \mathbf{E}(\mathbf{r}, \omega)=4 \pi \rho(\mathbf{r}, \omega), \\
\nabla \wedge \mathbf{B}(\mathbf{r}, \omega)=-\frac{i \omega}{c} \mathbf{E}(\mathbf{r}, \omega)+\frac{4 \pi}{c} \mathbf{j}(\mathbf{r}, \omega) .
\end{gathered}
$$

It is well known that all far- and near-field optical phenomena are contained in these four equations. Nevertheless, when dealing with the complex optical geometries currently investigated in NFO, the solution of these universal equations needs some specific care. Recently, we have demonstrated how the numerical difficulties inherent to the low symmetry of the subwavelength objects may be overcome by solving directly the integral equation associated with these equations in direct space. ${ }^{11,31,12,15,13}$ In the following subsection, we give a short survey of this technique.

\section{B. Integral equation for the optical electric field}

The vectorial wave equation for the electric field is readily obtained by taking the curl of Eq. (1). After some straightforward algebra, one gets the well-known result

$$
\Delta \mathbf{E}(\mathbf{r}, \omega)+k_{0}^{2} \mathbf{E}(\mathbf{r}, \omega)=4 \pi \nabla \rho(\mathbf{r}, \omega)-i k_{0} \frac{4 \pi}{c} \mathbf{j}(\mathbf{r}, \omega),
$$

where $k_{0}=\omega / c$ represents the wave vector associated to the frequency $\omega$.

We express now both charge and current densities in terms of the local polarization $\mathbf{P}(\mathbf{r}, \omega)$ of the material system:

$$
\rho(\mathbf{r}, \omega)=-\nabla \cdot \mathbf{P}(\mathbf{r}, \omega)
$$

and

$$
\mathbf{j}(\mathbf{r}, \omega)=-i \omega \mathbf{P}(\mathbf{r}, \omega) .
$$

We now rewrite the nonhomogeneous equation (5) as

$$
\Delta \mathbf{E}(\mathbf{r}, \omega)+k_{0}^{2} \mathbf{E}(\mathbf{r}, \omega)=-4 \pi\left\{\nabla[\nabla \cdot \mathbf{P}(\mathbf{r}, \omega)]+k_{0}^{2} \mathbf{P}(\mathbf{r}, \omega)\right\} .
$$

This differential equation can be converted into its integral form by using the standard Green's-function technique and the usual constitutive relation between the electric polarization inside the surface defect and the local electric field $\mathbf{E}(\mathbf{r}, \omega)$. This leads to

$$
\mathbf{E}(\mathbf{r}, \omega)=\mathbf{E}_{0}(\mathbf{r}, \omega)+\int_{v} \mathbf{S}_{0}\left(\mathbf{r}, \mathbf{r}^{\prime}, \omega\right) \chi\left(\mathbf{r}^{\prime}, \omega\right) \mathbf{E}\left(\mathbf{r}^{\prime}, \omega\right) d \mathbf{r}^{\prime},
$$

where the integral runs over the volume occupied by the surface defect. In Eq. (9) $\mathbf{E}_{0}(\mathbf{r}, \omega)$ represents the solution of the homogeneous equation (for example, the incident optical electric field) and $\chi\left(\mathbf{r}^{\prime}, \omega\right)$ is the linear electric susceptibility of the surface defect.

$\mathbf{S}_{0}\left(\mathbf{r}, \mathbf{r}^{\prime}, \omega\right)$ is the free-space dyadic propagator (also called electric field susceptibility), which can be found to be

$$
\mathbf{S}_{0}\left(\mathbf{r}, \mathbf{r}^{\prime}, \omega\right)=\left(k_{0}^{2}+\nabla \nabla\right) \mathcal{G}_{0}\left(\mathbf{r}, \mathbf{r}^{\prime}, \omega\right),
$$

where the scalar Green's function $\mathcal{G}_{0}\left(\mathbf{r}, \mathbf{r}^{\prime}, \omega\right)$ has the form of a spherical wave:

$$
\mathcal{G}_{0}\left(\mathbf{r}, \mathbf{r}^{\prime}, \omega\right)=\frac{e^{i k_{0}\left|\mathbf{r}-\mathbf{r}^{\prime}\right|}}{\left|\mathbf{r}-\mathbf{r}^{\prime}\right|}
$$

The integral equation (9) is very general. Indeed, if we introduce an additional perturbation due to, for example, the presence of a semi-infinite surface supporting the localized defect [characterized here by its optical response $\chi(\mathbf{r}, \omega)$, we only need to replace the free-space dyadic $\mathbf{S}_{0}\left(\mathbf{r}, \mathbf{r}^{\prime}, \omega\right)$ by the following one

$$
\mathbf{S}\left(\mathbf{r}, \mathbf{r}^{\prime}, \omega\right)=\mathbf{S}_{0}\left(\mathbf{r}, \mathbf{r}^{\prime}, \omega\right)+\mathbf{S}_{\mathrm{s}}\left(\mathbf{r}, \mathbf{r}^{\prime}, \omega\right),
$$

where the additional contribution $\mathbf{S}_{\mathrm{s}}\left(\mathbf{r}, \mathbf{r}^{\prime}, \omega\right)$ accounts for the dynamical response of the plane surface.

Before extending this procedure to the calculation of the magnetic near field and discussing various efficient numerical procedures to solve the resulting integral equation, let us 
recall that such response functions reveal how a dipolar source field is modified at the proximity of the surface limiting this system. This dyadic tensor has already been defined in the literature. For example, its retarded form is given in the Appendix of Ref. 16.

\section{Integral equation for the optical magnetic field}

Applying the same steps for the magnetic field equation (4), we can write

$$
\nabla \wedge[\nabla \wedge \mathbf{B}(\mathbf{r}, \omega)]=-i k_{0} \nabla \wedge \mathbf{E}(\mathbf{r}, \omega)+\frac{4 \pi}{c} \nabla \wedge \mathbf{j}(\mathbf{r}, \omega)
$$

Using then some standard vector analysis procedure, we can progress further,

$$
\nabla[\nabla \cdot \mathbf{B}(\mathbf{r}, \omega)]-\Delta \mathbf{B}(\mathbf{r}, \omega)=k_{0}^{2} \mathbf{B}(\mathbf{r}, \omega)+\frac{4 \pi}{c} \nabla \wedge \mathbf{j}(\mathbf{r}, \omega),
$$

which leads to

$$
\Delta \mathbf{B}(\mathbf{r}, \omega)+k_{0}^{2} \mathbf{B}(\mathbf{r}, \omega)=-\frac{4 \pi}{c} \nabla \wedge \mathbf{j}(\mathbf{r}, \omega)
$$

Let us note that, in the absence of any optical magnetic susceptibilities inside the perturbation, the source term occurring in this last equation is just proportional to the electrical polarization $\mathbf{P}(\mathbf{r}, \omega)$. Substituting Eq. (7) into Eq. (15), leads to

$$
\Delta \mathbf{B}(\mathbf{r}, \omega)+k_{0}^{2} \mathbf{B}(\mathbf{r}, \omega)=4 \pi i k_{0} \nabla \wedge \mathbf{P}(\mathbf{r}, \omega)
$$

According to a standard procedure in mathematical textbooks, we can solve this partial differential equation by adding to the solution of the homogeneous equation

$$
\Delta \mathbf{B}_{0}(\mathbf{r}, \omega)+k_{0}^{2} \mathbf{B}_{0}(\mathbf{r}, \omega)=0,
$$

a particular solution $\mathbf{B}_{\mathrm{m}}(\mathbf{r}, \omega)$ of the complete equation. Thanks to the Green's-functions technique already used to solve the electric field, one finds that this additional contribution originates from the current density source term. After some algebra, ones obtains

$$
\mathbf{B}_{\mathrm{m}}(\mathbf{r}, \omega)=-i k_{0} \int_{v} \nabla_{\mathbf{r}} \mathcal{G}_{0}\left(\mathbf{r}, \mathbf{r}^{\prime}, \omega\right) \wedge \mathbf{P}\left(\mathbf{r}^{\prime}, \omega\right) d \mathbf{r}^{\prime}
$$

We now have all the ingredients needed to write the general solution

$$
\mathbf{B}(\mathbf{r}, \omega)=\mathbf{B}_{0}(\mathbf{r}, \omega)-i k_{0} \int_{v} \nabla_{\mathbf{r}} \mathcal{G}_{0}\left(\mathbf{r}, \mathbf{r}^{\prime}, \omega\right) \wedge \mathbf{P}\left(\mathbf{r}^{\prime}, \omega\right) d \mathbf{r}^{\prime}
$$

At this stage, in the same way as it was done with the electric field, it is worthwhile to rewrite this equation in terms of the field propagator. Moreover, by applying the usual linear relation between electric polarization and electric field, we get the following general result:

$$
\mathbf{B}(\mathbf{r}, \omega)=\mathbf{B}_{0}(\mathbf{r}, \omega)+\int_{v} \mathbf{Q}_{0}\left(\mathbf{r}, \mathbf{r}^{\prime}, \omega\right) \chi\left(\mathbf{r}^{\prime}, \omega\right) \mathbf{E}\left(\mathbf{r}^{\prime}, \omega\right) d \mathbf{r}^{\prime},
$$

with

$$
\mathbf{Q}_{0}\left(\mathbf{r}, \mathbf{r}^{\prime}, \omega\right)=\left(\begin{array}{ccc}
0 & -\nabla_{z} \mathcal{G}_{0}\left(\mathbf{r}, \mathbf{r}^{\prime}, \omega\right) & \nabla_{y} \mathcal{G}_{0}\left(\mathbf{r}, \mathbf{r}^{\prime}, \omega\right) \\
\nabla_{z} \mathcal{G}_{0}\left(\mathbf{r}, \mathbf{r}^{\prime}, \omega\right) & 0 & \nabla_{x} \mathcal{G}_{0}\left(\mathbf{r}, \mathbf{r}^{\prime}, \omega\right) \\
-\nabla_{y} \mathcal{G}_{0}\left(\mathbf{r}, \mathbf{r}^{\prime}, \omega\right) & \nabla_{x} \mathcal{G}_{0}\left(\mathbf{r}, \mathbf{r}^{\prime}, \omega\right) & 0
\end{array}\right)
$$

This tensor can be elaborated further by using the free-space Green's function [cf. Eq. (11)]. In fact, after derivation, $\mathbf{Q}_{0}\left(\mathbf{r}, \mathbf{r}^{\prime}, \omega\right)$ can be split into two contributions: a far-field term $\mathbf{Q}_{0}^{(\text {far })}\left(\mathbf{r}, \mathbf{r}^{\prime}, \omega\right)$ and a near-field term $\mathbf{Q}_{0}^{(\text {near })}\left(\mathbf{r}, \mathbf{r}^{\prime}, \omega\right)$.

The first contribution with a global spatial variation proportional to $r^{-1}$ is given by

$$
\mathbf{Q}_{0}^{(\mathrm{far})}\left(\mathbf{r}, \mathbf{r}^{\prime}, \omega\right)=\frac{e^{i k_{0}\left|\mathbf{r}-\mathbf{r}^{\prime}\right|}}{\left|\mathbf{r}-\mathbf{r}^{\prime}\right|^{2}}\left(\begin{array}{ccc}
0 & -k_{0}^{2}\left(z-z^{\prime}\right) & k_{0}^{2}\left(y-y^{\prime}\right) \\
k_{0}^{2}\left(z-z^{\prime}\right) & 0 & -k_{0}^{2}\left(x-x^{\prime}\right) \\
-k_{0}^{2}\left(y-y^{\prime}\right) & k_{0}^{2}\left(x-x^{\prime}\right) & 0
\end{array}\right) \text {. }
$$

The near-field contribution varies with $r^{-2}$. It may be deduced from Eq. (21): 


$$
\mathbf{Q}_{0}^{(\text {near })}\left(\mathbf{r}, \mathbf{r}^{\prime}, \omega\right)=\frac{e^{i k_{0}\left|\mathbf{r}-\mathbf{r}^{\prime}\right|}}{\left|\mathbf{r}-\mathbf{r}^{\prime}\right|^{3}}\left(\begin{array}{ccc}
0 & -i k_{0}\left(z-z^{\prime}\right) & i k_{0}\left(y-y^{\prime}\right) \\
i k_{0}\left(z-z^{\prime}\right) & 0 & -i k_{0}\left(x-x^{\prime}\right) \\
-i k_{0}\left(y-y^{\prime}\right) & i k_{0}\left(x-x^{\prime}\right) & 0
\end{array}\right) \text {. }
$$

The two different powers $r^{-1}$ and $r^{-2}$ explain that $\mathbf{Q}_{0}^{(\mathrm{far})}\left(\mathbf{r}, \mathbf{r}^{\prime}, \omega\right)$ is dominating in the far field. This first contribution is thus related to the energy radiated far away from the sample, while $\mathbf{Q}_{0}^{(\text {near) }}\left(\mathbf{r}, \mathbf{r}^{\prime}, \omega\right)$ is responsible for most of the confinement of the optical magnetic field around subwavelength dielectric structures.

Before discussing some numerical strategies able to solve simultaneously the two integral equations (9) and (20), it is important to comment on these first analytical results.

(i) First, it is obvious from Eqs. (20) and (23) that even a free magnetic susceptibility dielectric structure is able to deeply modify the optical magnetic field distribution in the near-field zone.

(ii) Second, the magnitude of this effect depends linearly on the self-consistent optical electric-field distribution existing inside the dielectric material [cf. Eq. (20)]. Consequently, from a computational point of view, very stable solutions for the vector fields $\mathbf{B}(\mathbf{r}, \omega)$ can be deduced from the knowledge of the vector fields $\mathbf{E}(\mathbf{r}, \omega)$ inside the surface defect.

(iii) Third, in the near-field region the optical magnetic topography will be governed mainly by the symmetry properties of the propagator $\mathbf{Q}_{0}^{(\text {near) }}\left(\mathbf{r}, \mathbf{r}^{\prime}, \omega\right)$. This dyadic tensor, with its simple analytical form, will then be a precious tool to properly interpret numerical outputs.

(iv) Finally, we have to mention that in the same manner as for the electric field equation it is a simple matter to enlarge the application range of Eq. (20) by introducing a reference system different from vacuum. Indeed, if we want to introduce a somewhat more complicated surrounding located in the vicinity of the surface defect under study, we just need to replace the tensor $\mathbf{Q}_{0}\left(\mathbf{r}, \mathbf{r}^{\prime}, \omega\right)$ by

$$
\mathbf{Q}\left(\mathbf{r}, \mathbf{r}^{\prime}, \omega\right)=\mathbf{Q}_{0}\left(\mathbf{r}, \mathbf{r}^{\prime}, \omega\right)+\mathbf{Q}_{s}\left(\mathbf{r}, \mathbf{r}^{\prime}, \omega\right),
$$

where the additional contribution $\mathbf{Q}_{s}\left(\mathbf{r}, \mathbf{r}^{\prime}, \omega\right)$ accounts for the dynamical response of the dielectric surrounding. An example is detailed in the Appendix.

\section{GENERALIZED PROPAGATOR AND REAL-SPACE VOLUME DISCRETIZATION SCHEME}

\section{A. Analytical solution procedure}

As demonstrated in Sec. II, solving Eq. (20) requires the solution of Eq. (9) inside the surface defect. To realize this first step, we can use the generalized field propagator technique described in Ref. 15. This method enables us to transform the implicit Lippmann-Schwinger equation (9) into the following explicit integral relation:

$$
\mathbf{E}(\mathbf{r}, \omega)=\int_{v} \mathcal{K}\left(\mathbf{r}, \mathbf{r}^{\prime}, \omega\right) \cdot \mathbf{E}_{0}\left(\mathbf{r}^{\prime}, \omega\right) d \mathbf{r}^{\prime}
$$

As detailed in Ref. 15 , the dyadic $\mathcal{K}\left(\mathbf{r}, \mathbf{r}^{\prime}, \omega\right)$, also called generalized electric field propagator, can be expressed in terms of the optical field susceptibility tensor $\mathcal{S}\left(\mathbf{r}, \mathbf{r}^{\prime}, \omega\right)$ associated with the entire system (localized surface defect plus surrounding):

$$
\mathcal{K}\left(\mathbf{r}, \mathbf{r}^{\prime}, \omega\right)=\delta\left(\mathbf{r}-\mathbf{r}^{\prime}\right)+\mathcal{S}\left(\mathbf{r}, \mathbf{r}^{\prime}, \omega\right) \chi\left(\mathbf{r}^{\prime}, \omega\right) .
$$

The dyadic tensor $\mathcal{S}\left(\mathbf{r}, \mathbf{r}^{\prime}, \omega\right)$ in this equation can be derived numerically by using the Dyson's equation

$\mathcal{S}\left(\mathbf{r}, \mathbf{r}^{\prime}, \omega\right)=\mathbf{S}\left(\mathbf{r}, \mathbf{r}^{\prime}, \omega\right)+\int_{v} \mathbf{S}\left(\mathbf{r}, \mathbf{r}^{\prime}, \omega\right) \chi\left(\mathbf{r}^{\prime}, \omega\right) \mathcal{S}\left(\mathbf{r}, \mathbf{r}^{\prime}, \omega\right) d \mathbf{r}^{\prime}$.

As already discussed in previous published works, ${ }^{31,11,12,15}$ such developments performed in the real space instead of the reciprocal space, for both low-symmetry and lowdimensional systems, are well suited to cope with complex geometries. Furthermore, the numerical procedure derived from this methodology (see Ref. 15) has proven to be very stable even with large-scale computational systems. Backsubstitution of Eq. (25) into Eq. (20) then yields a general solution for the optical magnetic field. In a consistent way with the linear response hypothesis introduced above, this solution is linearly related to both incident electric $\mathbf{E}_{0}(\mathbf{r}, \omega)$ and magnetic fields $\mathbf{B}_{0}(\mathbf{r}, \omega)$ :

$$
\begin{aligned}
\mathbf{B}(\mathbf{r}, \omega)= & \mathbf{B}_{0}(\mathbf{r}, \omega)+\iint_{v} \mathbf{Q}\left(\mathbf{r}, \mathbf{r}^{\prime}, \omega\right) \chi\left(\mathbf{r}^{\prime}, \omega\right) \\
& \times \mathcal{K}\left(\mathbf{r}^{\prime}, \mathbf{r}^{\prime \prime}, \omega\right) \mathbf{E}_{0}\left(\mathbf{r}^{\prime \prime}, \omega\right) d \mathbf{r}^{\prime} d \mathbf{r}^{\prime \prime}
\end{aligned}
$$

At this stage it may be worthwhile to note that, in the same manner as we have done with the optical electric field [cf. Eq. (25)], we have also the opportunity to define from Eq. (28) a mixed generalized propagator able to couple electric and magnetic field distribution. Using Eq. (4), we can write

$$
\mathbf{B}(\mathbf{r}, \omega)=\int_{v} \mathcal{L}\left(\mathbf{r}, \mathbf{r}^{\prime}, \omega\right) \cdot \mathbf{E}_{0}\left(\mathbf{r}^{\prime}, \omega\right) d \mathbf{r}^{\prime} .
$$

The dyadic operator $\mathcal{L}\left(\mathbf{r}, \mathbf{r}^{\prime}, \omega\right)$ is defined by

$$
\begin{aligned}
\mathcal{L}\left(\mathbf{r}, \mathbf{r}^{\prime}, \omega\right)= & \frac{\delta\left(\mathbf{r}-\mathbf{r}^{\prime}\right)}{i k_{0}} \Lambda_{\mathbf{r}^{\prime}} \\
& +\int_{v} \mathbf{Q}\left(\mathbf{r}, \mathbf{r}^{\prime}, \omega\right) \chi\left(\mathbf{r}^{\prime \prime}, \omega\right) \mathcal{K}\left(\mathbf{r}^{\prime \prime}, \mathbf{r}^{\prime}, \omega\right) d \mathbf{r}^{\prime \prime},
\end{aligned}
$$

where $\Lambda_{\mathbf{r}^{\prime}}$ labels the matrix form of the curl operator. The useful property of $\mathcal{L}\left(\mathbf{r}, \mathbf{r}^{\prime}, \omega\right)$ is that it only depends on the geometry of the scattering system; it does not depend on the incident electric field. 


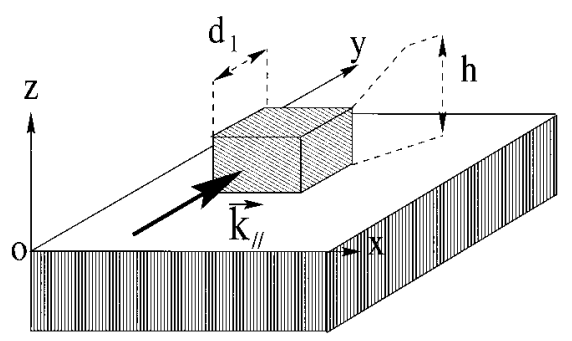

FIG. 1. Perspective drawing of a square-shaped subwavelength surface defect lying on a flat surface. Defect and support have the same optical index $(n=1.458)$. The system is illuminated in total internal reflection and the incident wavelength in vacuum is equal to $633 \mathrm{~nm}$. The object height is $h=40 \mathrm{~nm}$ and the side of its square section $d_{1}=100 \mathrm{~nm} ; \mathbf{k}_{\|}$represents the surface wave vector associated with the excitation field.

\section{B. Numerical method}

Although Eq. (29) establishes a direct relation between $\mathbf{B}(\mathbf{r}, \omega)$ and $\mathbf{E}_{0}(\mathbf{r}, \omega)$, for computational purpose, we prefer starting with the less elaborate Eq. (28). In scattering theory, the first term $\mathbf{B}_{0}(\mathbf{r}, \omega)$ is referred to as the incident field while the second term is called the scattered field obtained from the integration over the domain $v$ where $\chi\left(\mathbf{r}^{\prime}, \omega\right)$ is nonzero. In the present study, $v$ defines the volume of the localized surface defect supported by a plane dielectric sample (an example is depicted in Fig. 1). Electromagnetic theory traditionally qualifies $v$ as the source region. ${ }^{32} \mathrm{We}$ will discuss in this subsection how the discretization of Eq. (28) allows one to obtain the numerical outputs for the magnetic field outside the source region. For an isotropic and homogeneous surface defect of dielectric constant $\epsilon(\omega)$, such a procedure leads to
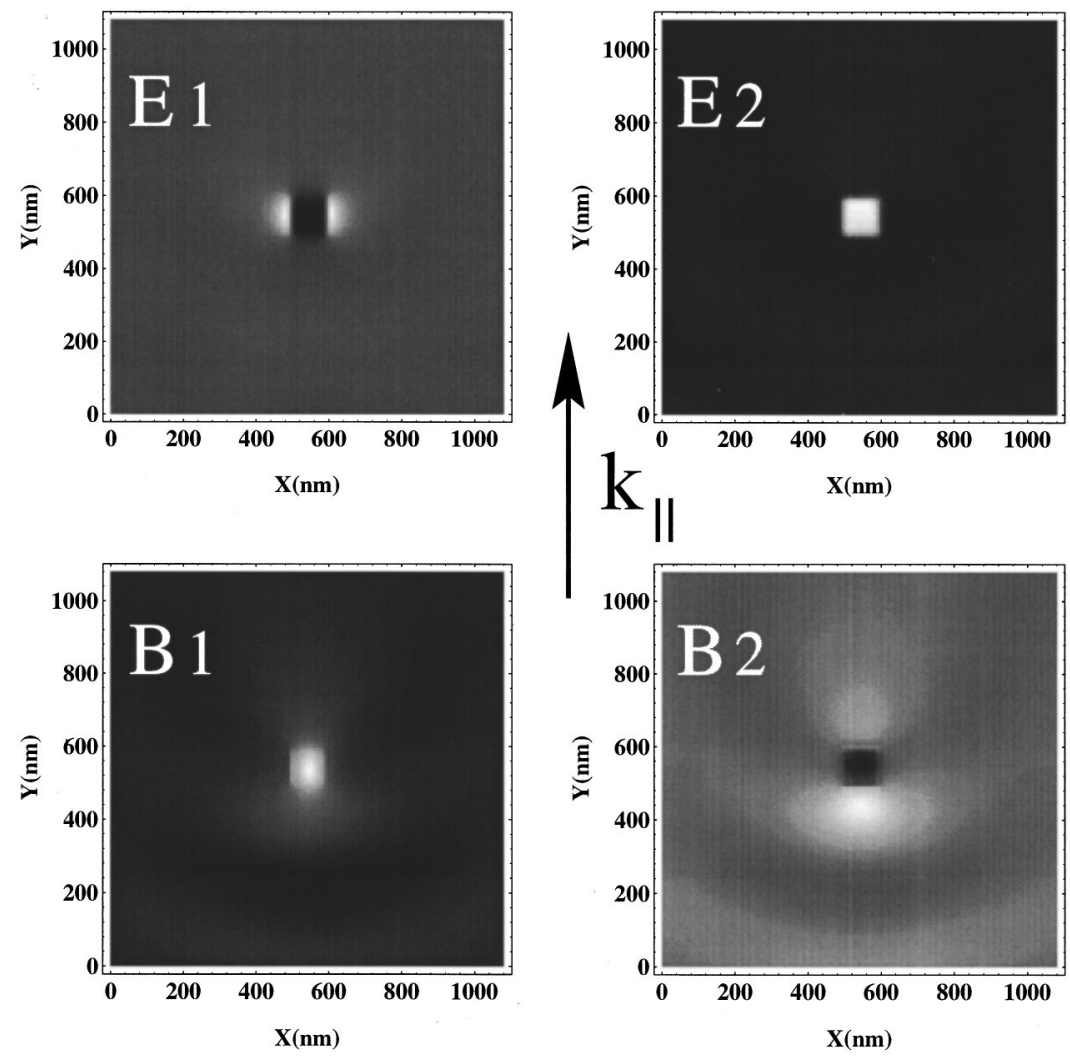

FIG. 2. Gray scale of both field intensities distributions $\left|\mathbf{E}\left(X, Y, Z_{0}\right)\right|^{2}$ and $\left|\mathbf{B}\left(X, Y, Z_{0}\right)\right|^{2}$ calculated above the topographic object described in Fig. 1. The intensity is computed in a plane parallel to the surface bearing the defect and located at a height $Z_{0}=50 \mathrm{~nm}$. The scanned area is $1080 \times 1080 \mathrm{~nm}^{2}$ and the incident wavelength $633 \mathrm{~nm}$. The two maps, labeled $(E 1)$ and $(E 2)$, describe the optical electric intensities $\left|\mathbf{E}\left(X, Y, Z_{0}\right)\right|^{2}$ computed respectively for the $s$ and $p$ polarized modes. The maps $(B 1)$ and (B2) represent the optical magnetic intensities $\left|\mathbf{B}\left(X, Y, Z_{0}\right)\right|^{2}$ calculated respectively for $s$ and $p$ polarized modes. 
optical indexes of both the protrusion and the supporting surface are equal to that of glass $(n=1.458)$, and all the numerical applications have been made with an incident angle $\theta_{\text {inc }}=60^{\circ}$.

Figure 2 presents four different near-field maps of the object depicted in Fig. 1. The intensities are computed in a plane parallel to the surface bearing the surface defect and located at a height $Z_{0}=50 \mathrm{~nm}$. The scanned area is $1080 \times 1080 \mathrm{~nm}^{2}$ and the incident wavelength $633 \mathrm{~nm}$. The analysis of these results raises the following comments.

(i) The two first maps, labeled $(E 1)$ and $(E 2)$, describe the optical electric intensities $\left|\mathbf{E}^{2}\left(X, Y, Z_{0}\right)\right|$ computed in the $s$ and $p$ polarized modes, respectively. As already predicted from previous numerical simulations, while $p$-polarized surface waves lead to a strong confinement of the total electric field intensity above the surface defects, $s$ polarization gives rise to large electric field intensity gradients at the vicinity of the pads with the well-known dark contrast phenomena recently observed in near-field optical microscopy. ${ }^{18}$ These effects are consistent with previous calculations performed near 3D surface protrusions. ${ }^{16}$

(ii) The two last maps $(B 1)$ and $(B 2)$ gathered in Fig. 2 represent the optical magnetic intensities $\left|\mathbf{B}\left(X, Y, Z_{0}\right)\right|^{2}$ calculated with the same polarization states $(s$ and $p)$. In this case we observe a drastic change of the near-field image. The most impressive effect is the occurrence of a bright contrast when working with an $s$-polarized wave and an obvious dark contrast in the $p$-polarized case, always accompanied of two enhancements of the magnetic field just above the two edges perpendicular to the direction propagation ( $O X$ axis). As was done in the case of the electric field, ${ }^{16,18}$ the phenomenon of contrast reversal observed in Fig. 2(B2) can be simply explained by examining the structure of the dominating shortrange term $\mathbf{Q}_{0}^{(\text {near) }}\left(\mathbf{r}_{a}, \mathbf{r}_{0}\right)$ composing the free space mixed propagator $\mathbf{Q}_{0}$ [cf. Eq. (23)]. For this purpose, let us replace our isolated pad by a single system of polarizability $\alpha_{a}(\omega)$ located at the position $\mathbf{r}_{a}=\left(0,0, z_{a}\right)$. Applying then the first
Born approximation to Eq. (20), the magnetic near field spawned by the object reduces to

$$
\mathbf{B}(\mathbf{r}, \omega) \simeq \mathbf{B}_{0}(\mathbf{r}, \omega)+\alpha_{a}(\omega) \mathbf{Q}_{0}^{\text {near }}\left(\mathbf{r}-\mathbf{r}_{a}, \omega\right) \mathbf{E}_{0}\left(\mathbf{r}_{a}, \omega\right),
$$

where for the $p$-polarized mode we have

$$
\begin{gathered}
E_{0 x}(\mathbf{r}, \omega)=0, \\
E_{0 y}(\mathbf{r}, \omega)=A_{0} T_{p} \delta_{c}, \\
E_{0 z}(\mathbf{r}, \omega)=A_{0} T_{p} \delta_{s},
\end{gathered}
$$

and

$$
\begin{gathered}
B_{0 x}(\mathbf{r}, \omega)=-A_{0} T_{p} \sin \left(\theta_{\mathrm{c}}\right), \\
B_{0 y}(\mathbf{r}, \omega)=0, \\
B_{0 z}(\mathbf{r}, \omega)=0,
\end{gathered}
$$

with

$$
\begin{gathered}
\delta_{s}=\frac{\sin \left(\theta_{\text {inc }}\right)}{\sin \left(\theta_{\mathrm{c}}\right)}, \\
\delta_{c}=\frac{i\left[\sin ^{2}\left(\theta_{\mathrm{inc}}\right)-\sin ^{2}\left(\theta_{\mathrm{c}}\right)\right]^{1 / 2}}{\sin \left(\theta_{\mathrm{c}}\right)} .
\end{gathered}
$$

In Eqs. (33) and (34), $A_{0}$ is a scalar parameter proportional to $\exp \left\{-i k_{\|} y-\left[\sin ^{2}\left(\theta_{\text {inc }}\right)-\sin ^{2}\left(\theta_{\mathrm{c}}\right)\right]^{1 / 2} z\right\}$, where $\theta_{c}$ represents the critical angle for total reflection of the material, and the factor $T_{p}$ is the usual transmission coefficient for $p$ polarization. Finally from Eqs. (23), (32), (33), and (34), we can easily verify that when the observation point $\mathbf{r}$ passes just above the surface protrusion [i.e., when $\left.\mathbf{r}=\left(0,0, Z_{0}\right)\right]$, Eq. (32) produces a total magnetic field directed along the $O X$ axis

$$
\mathbf{B}(\mathbf{r}, \omega) \simeq\left\{-A_{0} T_{p} \sin \left(\theta_{\mathrm{c}}\right)+\frac{A_{0} T_{p} k_{0} \alpha_{a}(\omega)}{\left(Z-z_{a}\right)^{2}} \frac{\left[\sin ^{2}\left(\theta_{\mathrm{inc}}\right)-\sin ^{2}\left(\theta_{\mathrm{c}}\right)\right]^{1 / 2}}{\sin \left(\theta_{\mathrm{c}}\right)}\right\} \mathbf{u}_{x},
$$

where $\mathbf{u}_{x}$ labels the unit vector associated with the $O X$ axis. The first term of this relation represents the value of the magnetic excitation field associated with the surface wave, while the second one accounts for the presence of the surface defect. When the incident angle $\theta_{\text {inc }}$ is greater than $\theta_{c}$, this additional contribution due the intrinsic form of the mixed propagator $\mathbf{Q}_{0}^{\text {(near) }}$ is always in the opposite direction with respect to the magnetic excitation field $\mathbf{B}_{0}$. Consequently, when the observation point is located on the top of the surface protrusion, we observe in this polarization mode a significant decrease of the total magnetic field intensity as predicted by the self-consistent calculation described in Fig. 2. Moreover, Eq. (36) indicates that $\mathbf{B}(\mathbf{R}, \omega)$ depends critically on the angle of incidence $\theta_{\text {inc. }}$. In the example reported in Fig. 2(B2), the magnetic intensity decreases by about $15 \%$ of the total intensity for an incident angle $\theta_{\text {inc }}=60^{\circ}$.

With this first simulation, we have proven that, first, the dielectric structures on a surface can produce a specific confinement of the optical magnetic field and, second, that the relation between the object profile and the resulting magnetic map depends strongly on the illumination mode. Let us now see what happens with the more elaborate localized surface structure described in Fig. 3. In this second application we have considered seven identical square-shaped pads and the four typical electric and magnetic intensity maps corresponding to this object are gathered in Figs. 4 and 5. Note that the electric maps (Fig. 4) are just given here for comparison purposes, because they were already discussed in a work published previously. ${ }^{16}$ In this application where each dielectric structure displays subwavelength lateral dimensions, the 


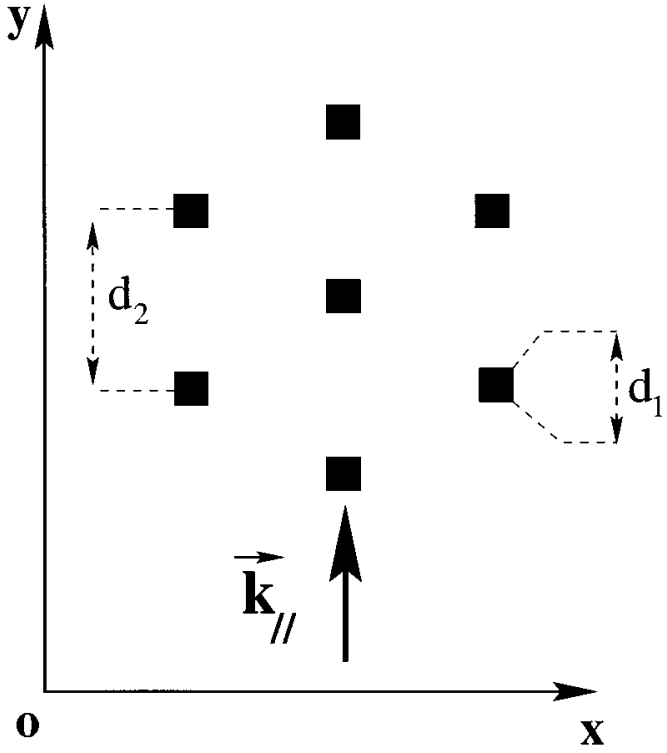

FIG. 3. Top view of a spatially localized dielectric system composed by seven identical square shaped protrusions lying on a perfectly plane surface. The center of each pad is located at the nodes of a hexagon with side $d_{2}$. The dimension of each individual protrusion is $d_{1}$ and $\mathbf{k}_{\|}$represents the projection of the incident wave vector on the surface $(X O Y)$. For the applications of Figs. 4, 5, and 6 , the following input parameters are used: $d_{1}=90 \mathrm{~nm}, d_{2}=375$ $\mathrm{nm}$, and the pads' height is $45 \mathrm{~nm}$.

magnetic intensity patterns display variations that are localized around the tops of the protrusions and slightly modulated by fringes due to a surface wave. Figure 6 shows two scan lines of these two magnetic maps, calculated along a line passing over the middle of three lined up pads. In the $p$-polarized mode (dashed line) we observed once again the contrast reversal phenomena predicted by the analytical Eq. (36).

\section{COMPARISON WITH NUMERICAL RESULTS ISSUED FROM THE DTG METHOD}

In this section, in order to assess the reliability of our numerical analysis, we report a careful comparison between magnetic field calculations performed with the DSIEM described above and with those issued from the DTG Maxwell's equation solver. By starting from the test object depicted in Fig. 1, we will discuss and compare the numerical solutions for different polarizations of the incident wave and different scan lines directions.

\section{A. The differential theory of gratings}

The complex problem related to the field distribution calculations near complex surface profiles or inside NFO devices $^{28}$ can also be investigated with theories based on diffraction gratings. ${ }^{24-26}$ The DTG method was originally developed twenty years ago to predict the efficiencies of oneand two-dimensional diffracting gratings. Based on a rigorous treatment of Maxwell's equations, this method can be also efficiently used to determine the optical near-field scattered by three-dimensional periodic samples. In the following subsection, in order to avoid a complete presentation of
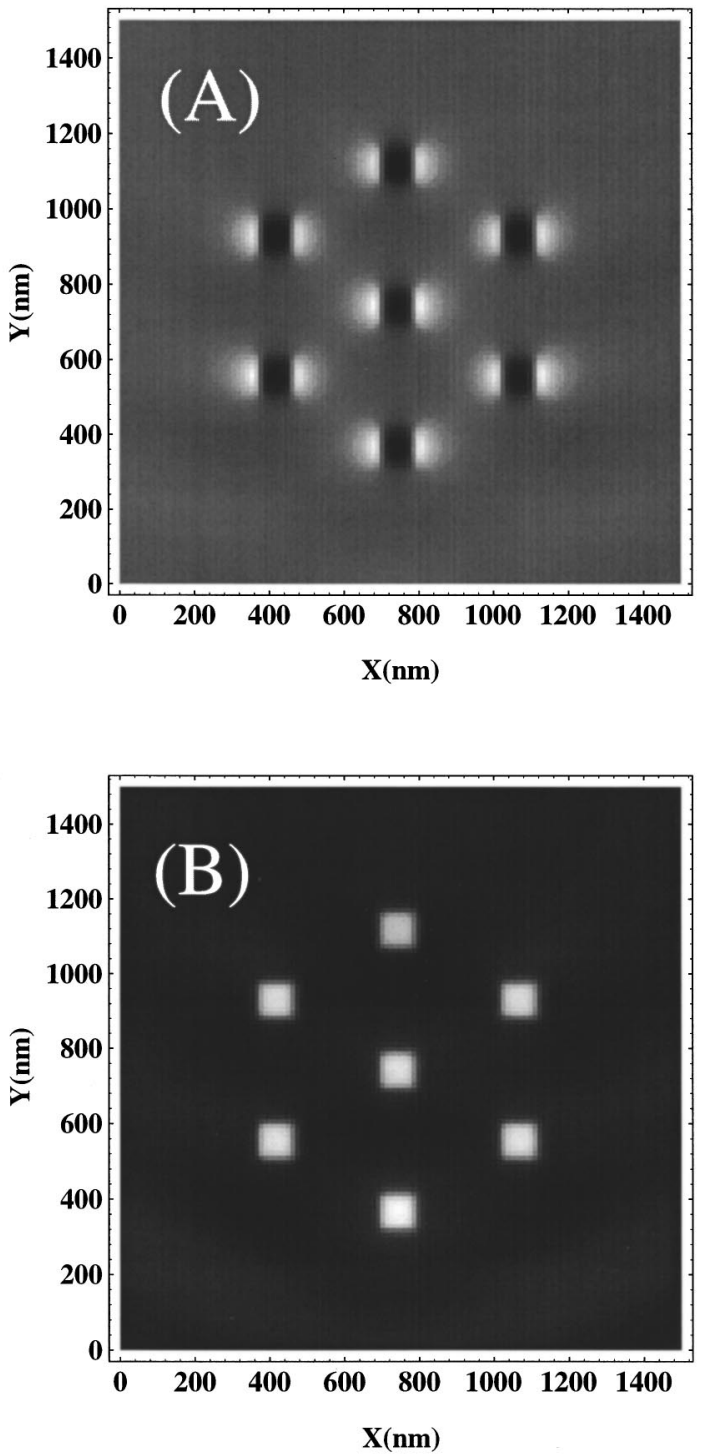

FIG. 4. Gray scale field distributions $\left|\mathbf{E}\left(X, Y, Z_{0}\right)\right|^{2}$ describing the evolution of the optical electric field around the topographic objects described in Fig. 3 (intensity growing from black to white). The same incident wavelength $\lambda=633 \mathrm{~nm}$ is considered for the two successive images and the observation plane is located at $58 \mathrm{~nm}$ from the pads' top. Two polarizations are considered: (a) $s$-polarized mode and (b) $p$-polarized mode.

this well-established technique, we will only summarize the essential steps of the computational procedure.

Basically, as exposed in previous sections, we are interested by the electromagnetic near-field diffracted above an object engraved on a flat glass-air interface illuminated by total internal reflection. When using the DTG method, ${ }^{24} \mathrm{a}$ nonperiodic isolated scattering object is viewed as an infinite diffracting grating built with a very large periodic spacing between each surface structure. Consequently, the first step in the calculation of the field diffracted by a nonperiodic sample is related to the choice of a period sufficiently large to prevent all overlapping effects between the near field spawned by each indivual pattern of the grating. The electromagnetic field diffracted above the grating can then be expanded in Fourier series 

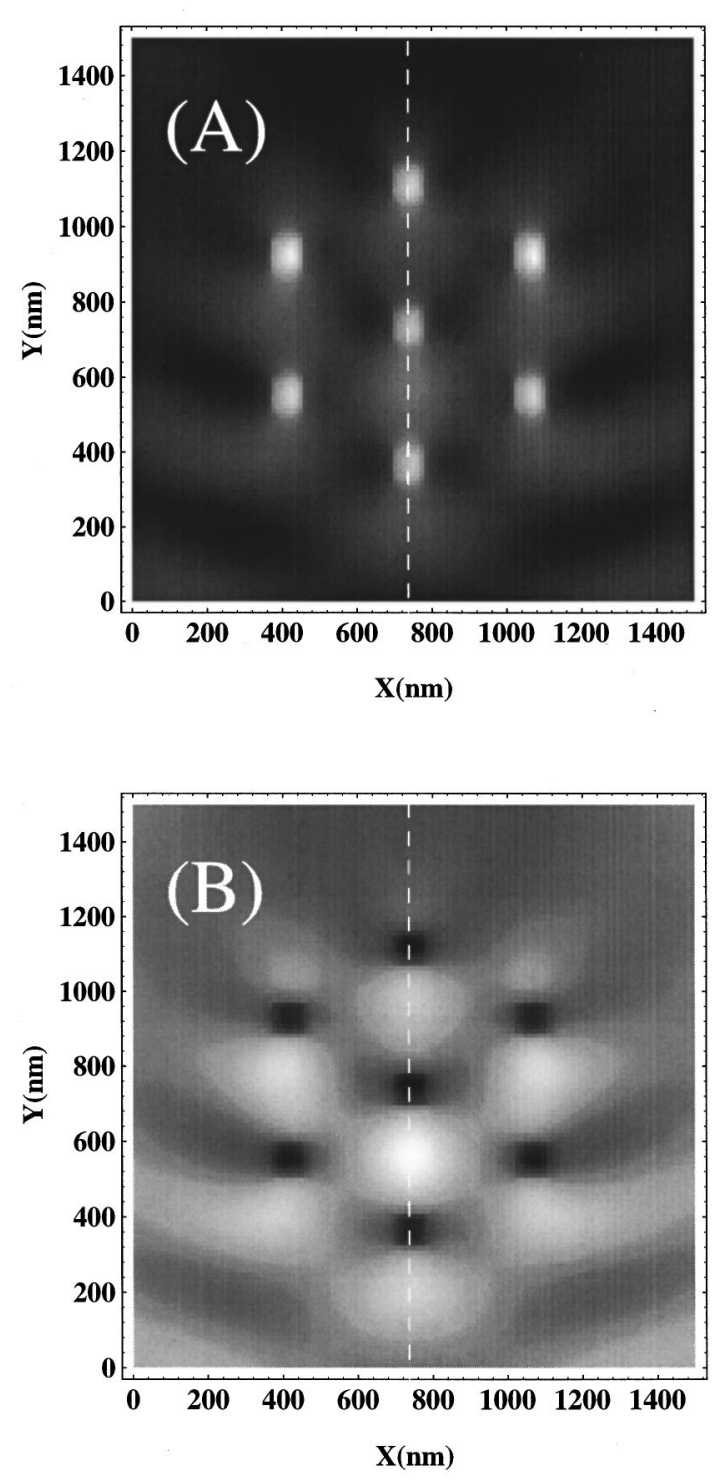

FIG. 5. Same situation as in Fig. 4, but for the optical magnetic field distribution $\left|\mathbf{B}\left(X, Y, Z_{0}\right)\right|^{2}$.

$$
\mathbf{A}(\mathbf{l}, z)=\sum_{r=-\infty}^{+\infty} \sum_{s=-\infty}^{+\infty} \mathbf{A}(r, s) e^{i \gamma(r, s) z} e^{i \mathbf{k} \|(r, s) \cdot \mathbf{l}},
$$

where $\mathbf{l}=(x, y), \mathbf{A}(\mathbf{l}, z)$ represents either the electric field $\mathbf{E}(\mathbf{l}, z)$ and the magnetic field $\mathbf{B}(\mathbf{l}, z)$. The 3D wave vectors $\mathbf{k}(r, s)=\left[\mathbf{k}_{\|}(r, s), \gamma(r, s)\right]$, associated with the harmonic $(r, s)$ obey the well-known dispersion equation

$$
\mathbf{k}_{\|}^{2}(r, s)+\gamma^{2}(r, s)=n^{2} k_{0}^{2} .
$$

The set of wave vector $\mathbf{k}_{\|}(r, s)$ parallel to the surface are simply defined for each couple of integer numbers $(r, s)$ by

$$
\mathbf{k}_{\|}(r, s)=\left(n k_{0 x}+r \frac{2 \pi}{d_{x}}\right) \mathbf{u}_{x}+\left(n k_{0 y}+s \frac{2 \pi}{d_{y}}\right) \mathbf{u}_{y},
$$

where $d_{x}$ and $d_{y}$ denote, respectively, the period of the grating along the $O X$ and $O Y$ directions. From Eq. (38), it may be seen that the coefficient $\gamma(r, s)$ may be either real or purely imaginary. The real values of $\gamma(r, s)$ correspond to

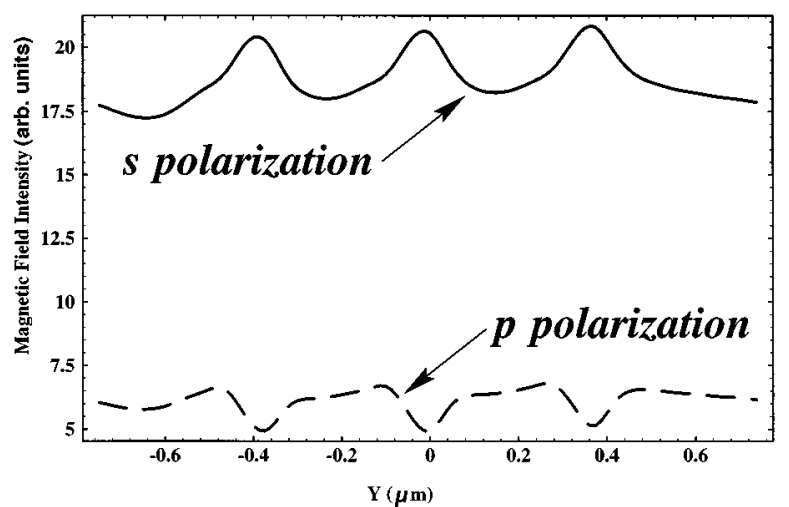

FIG. 6. Variation of the magnetic field intensity $\mid \mathbf{B}(X=750$ $\left.\mathrm{nm}, Y, Z_{0}\right)\left.\right|^{2}$ along a scan line parallel to the $O Y$ axis over the middle of three lined up dielectric pads. These cross sections, issued from the maps of Fig. 5, have been calculated for the same approach distance $Z_{0}=58 \mathrm{~nm}$. The solid and the dashed lines represent, respectively, $s$ - and $p$-polarized modes.

radiative harmonics while imaginary values introduce evanescent components in expansion (37).

In a general way, the six components of the electromagnetic field $\mathbf{A}(\mathbf{l}, z)$ can be deduced from a couple of independent parameters usually named the principal components. Let us choose, for example, the $Y$ components $E_{y}(\mathbf{l}, z)$ and
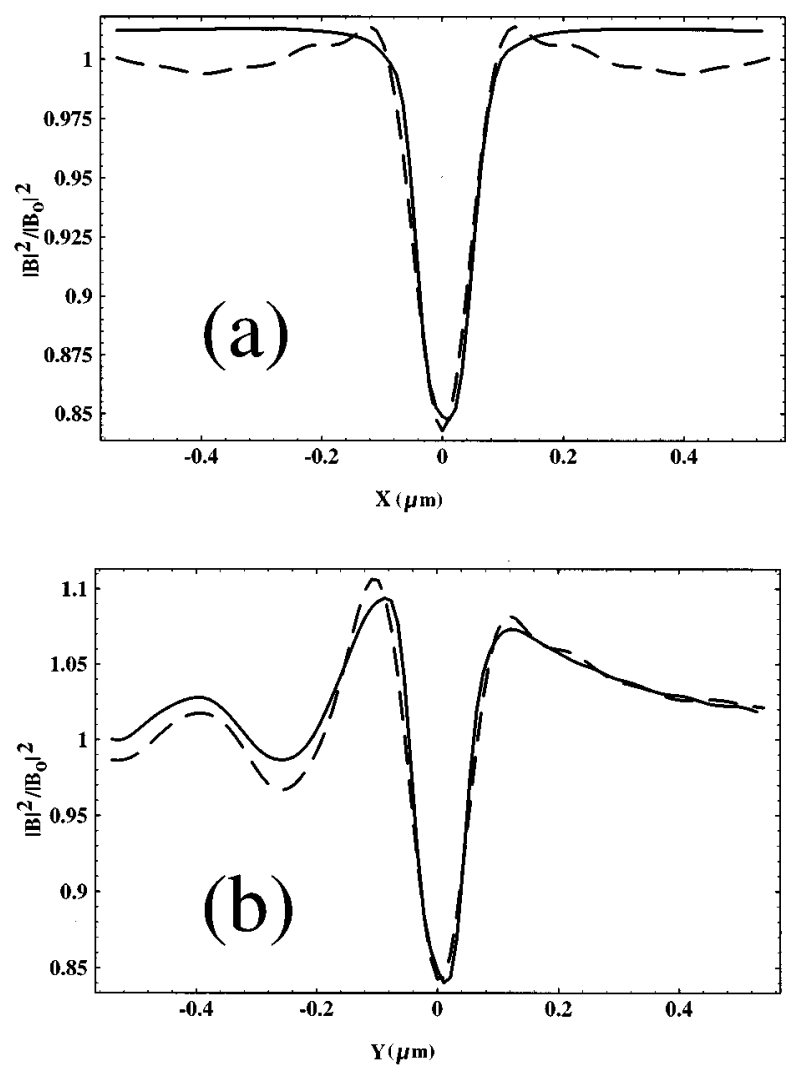

FIG. 7. Comparison of relative magnetic field intensity $\mathbf{B}^{2} / \mathbf{B}_{0}{ }^{2}$ scans obtained with the DSIEM (continuous line) and the DTG (dashed line) in the $s$-polarized mode. The scans are performed along the center of the square-shaped surface protrusion depicted in Fig. 1: (a) The calculation is performed along the $O X$ axis; (b) same calculation along the $O Y$ axis. 

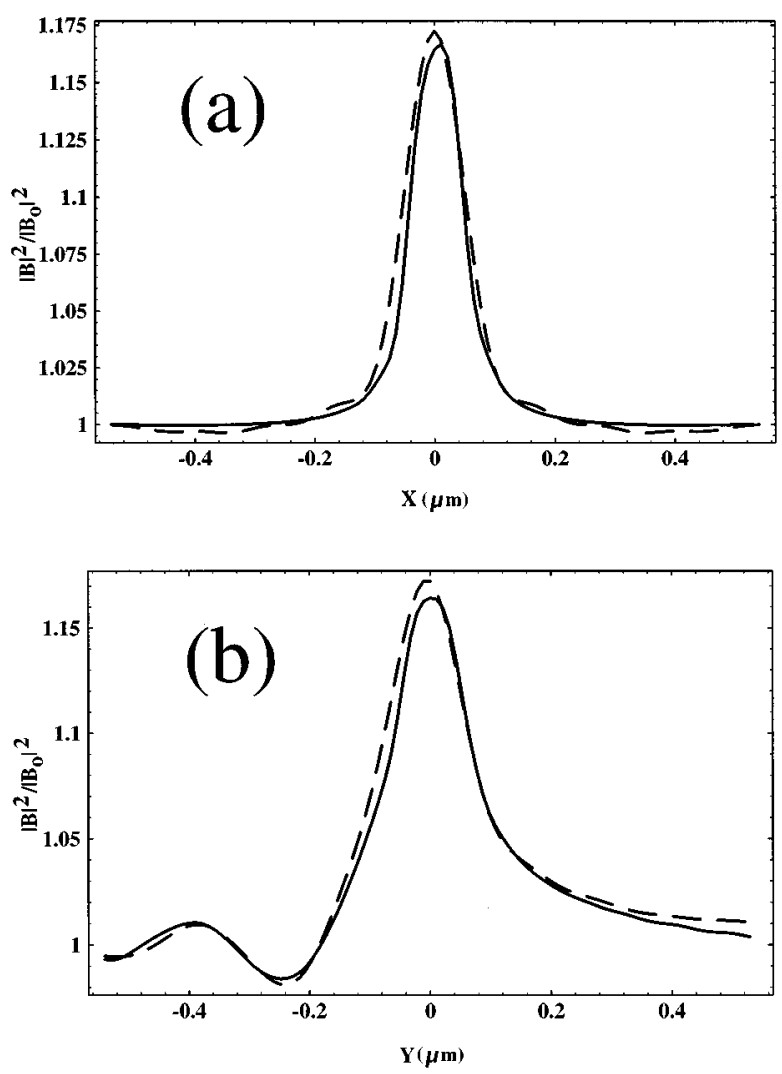

FIG. 8. Same as Fig. 7, but in $p$-polarized mode: (a) The calculation is performed along the $O X$ axis; (b) Same calculation along the $O Y$ axis.

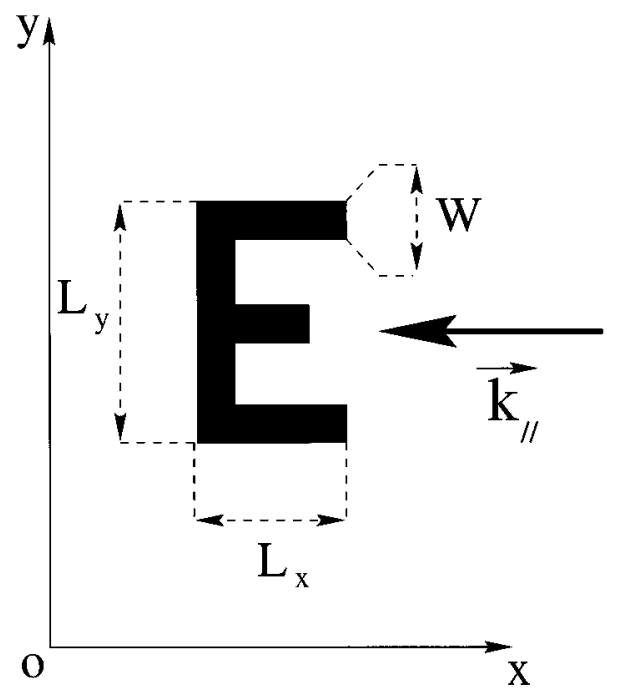

FIG. 9. Top view of the 3D dielectric pattern used in the simulation presented in Fig. 10. We have considered a letter $\mathbf{E}$ with a 40$\mathrm{nm}$ thickness and an index of refraction $n=1.458$ identical to that of the substrate. The other geometrical parameters are $W=50 \mathrm{~nm}$, $L=200 \mathrm{~nm}$, and $L_{y}=350 \mathrm{~nm}$. The projection of the incident wave vector on the $(X O Y)$ surface is represented by $\mathbf{k}_{\|}$.
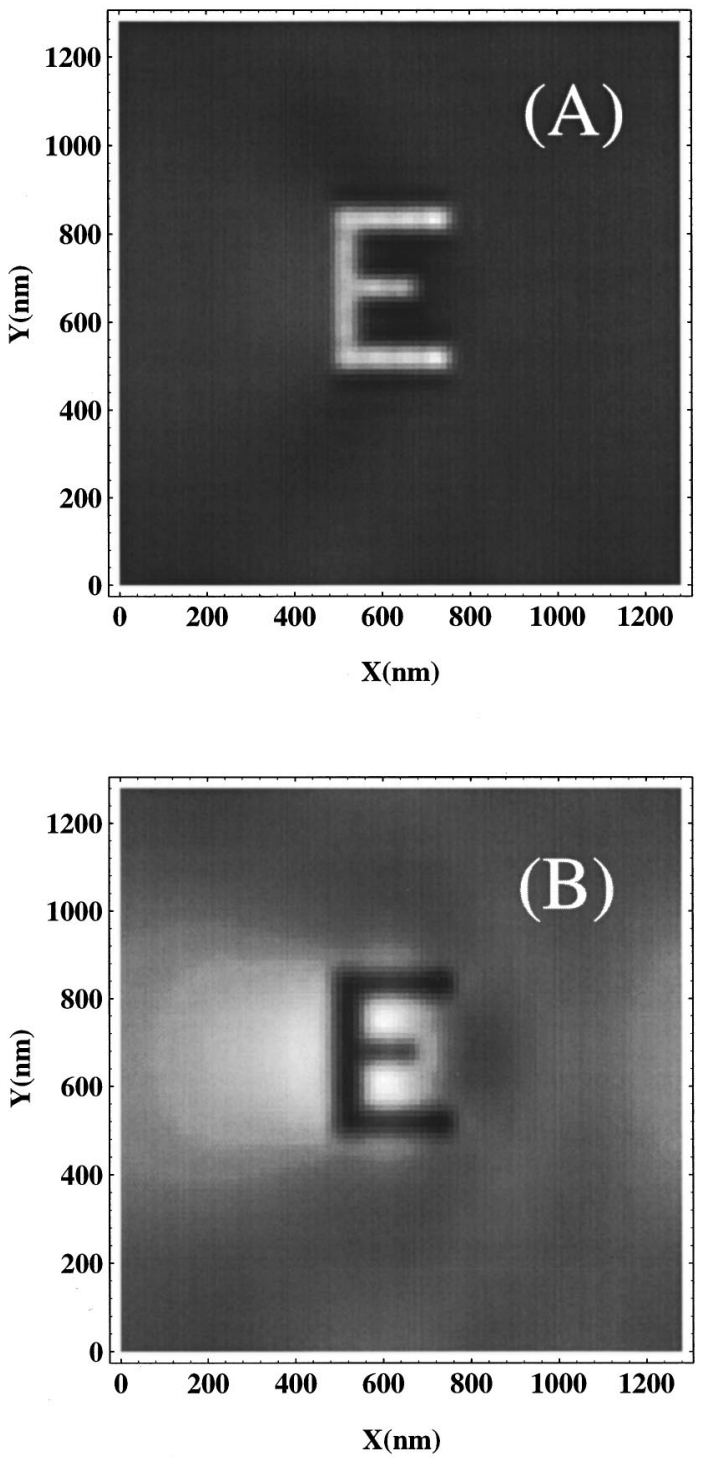

FIG. 10. Gray scale optical magnetic images calculated above the 3D object described in Fig. 9 from the DTG Maxwell's equation solver. The scanned area is $1250 \times 1250 \mathrm{~nm}^{2}$, the incident wavelength $633 \mathrm{~nm}$, and the calculation is performed at $50 \mathrm{~nm}$ from the flat sample. Two polarizations are considered: (a) $s$-polarized mode and (b) $p$-polarized mode.

$B_{y}(\mathbf{l}, z)$ as principal components. It is then a simple matter to show that their Fourier coefficients can be expressed as a linear combination of the $Y$ component of the incident field:

$$
\begin{aligned}
& E_{y}(r, s)=\mathcal{T}_{E}^{E}(r, s) E_{0 y}+\mathcal{T}_{B}^{E}(r, s) B_{0 y}, \\
& B_{y}(r, s)=\mathcal{T}_{E}^{B}(r, s) E_{0 y}+\mathcal{T}_{B}^{B}(r, s) B_{0 y} .
\end{aligned}
$$

The transmission coefficients $\mathcal{T}_{E}^{E}, \mathcal{T}_{B}^{E}, \mathcal{T}_{E}^{B}$, and $\mathcal{T}_{B}^{B}$ describe the coupling between the electric and magnetic harmonics composing the scattered and the incident field. These coefficients depend both on the geometry of the sample and on the angular conditions of incidence but not on the polarization of the incident light. The polarization of the incident plane wave is controlled by the values of $B_{0 y}$ and $E_{0 y}$. From a numerical point of view, the computation of the transmission 
coefficients begins with the inversion of a complex square matrix whose dimensions are $2 N_{T} \times 2 N_{T}$ [where $N_{T}$ is the total number of harmonics used to describe the scattered field in Eq. (37)]. A detailed description of the calculation of the matrix elements can be found in Refs. 24-26. If all the Fourier components of $E_{y}(r, s)$ and $B_{y}(r, s)$ are known, then all the components of the electromagnetic field can be calculated at each point located above the object. In a second step, the values of the field above the object can be used as initial conditions to integrate numerically Maxwell's equations in order to obtain the field inside the diffracting object. The propagation of this field outside the object provides simultaneously the numerical values of the electric and magnetic optical field anywhere.

\section{B. Comparison of the numerical solutions}

The cross check of the results obtained with different approaches is important because many fundamental near-field optical phenomena are extremely subtle and difficult to model. Therefore, in order to strengthen the reliability of our numerical analysis we propose in Figs. 7 and 8(a) a comparison of the relative total magnetic field intensity $|\mathbf{B}|^{2} /\left|\mathbf{B}_{0}\right|^{2}$ obtained with the DSIEM (continuous line) and the DTG (dashed line). The scans are performed along the center of the structure sketched in Fig. 1, at a height $Z_{0}=50 \mathrm{~nm}$ and normalized to the value $\left|\mathbf{B}_{0}\right|^{2}$ of the magnetic field intensity computed for a perfectly flat surface (without protrusions). We note the extremely good quantitative agreement between both methods. The small differences in the solution originates from residual collective effects generated by the infinite array of localized surface defects investigated with the DTG method.

For $s$ polarization, the increase of intensity occurring above the single pad is well restored by both methods (Fig. 7). For $p$ polarization, the magnetic field depletion is also well reproduced (Fig. 8). These last results emphasize the ability of these two methods to accurately reproduce subtle phenomena associated with optical magnetic confined fields. To conclude this section, we have tested the numerical stability of the DTG method on the very low symmetry object depicted in Fig. 9. For this last simulation, we have considered a letter $\mathbf{E}$ of $40 \mathrm{~nm}$ in thickness with an optical index of refraction $n=1.458$, identical to that of the substrate. The other geometrical parameters are $W=50 \mathrm{~nm}, L_{x}=200 \mathrm{~nm}$, $L_{y}=350 \mathrm{~nm}$.

The optical magnetic field maps resulting from the interaction of this object with a polarized surface wave is reported in Fig. 10. This figure emphasizes once again the influence of the illumination conditions on the imaging properties of such subwavelength objects.

\section{CONCLUSION}

Using scattering theory, we have investigated the distribution of the optical magnetic field around nanoscopic dielectric objects. Inspection of the analytical expression of the electromagnetic propagator shows that the magnetic field scattered by a dielectric object depends on the self-consistent electric field inside the object. It results that, in well-defined conditions of illumination that have been quantitatively analyzed in this paper, a nanoscopic dielectric structure without any magnetic property perturbs strongly the incident optical magnetic field.

Numerical simulations using two different computational methods indicate that a surface wave incident on a nanoscopic surface defect is scattered differently according to the incident polarization. The map of the optical magnetic nearfield intensity computed at constant height close above the surface defect exhibit a bright contrast in the $s$ polarization and a dark one in the $p$ polarization. These contrasts appear to be reversed compared to the ones observed for the optical electric near field. ${ }^{18}$

These results not only illustrate the properties of electromagnetic near field, but also may help to interpret near-field optical images that cannot be understood from the map of the electric near-field intensity. By these, we mean particularly many NFO experiments performed with dielectric probes coated with metal. For this aim, one should search the possible detection mechanism of optical magnetic near field with such a local probe.

\section{ACKNOWLEDGMENTS}

The Laboratoire de Physique Moléculaire is UMR CNRS 6624. The Laboratoire de Physique is UPRESA CNRS Q5027 and benefited from the financial support of the Region of Burgundy.

\section{APPENDIX: RETARDED ELECTRIC-MAGNETIC MIXED SUSCEPTIBILITY (EMMS) ASSOCIATED WITH A BARE PLANE DIELECTRIC SURFACE}

This mixed surface propagator converts the retarded response electric field of a fluctuating dipole moment into a magnetic response field. Consequently, it can be simply derived by taking the curl of the electric surface propagator $\mathbf{S}_{s}\left(\mathbf{r}, \mathbf{r}^{\prime}, \omega\right)$ available in the literature. In the case of a plane semi-infinite dielectric surface, such a calculation leads to

$$
\mathbf{Q}_{s}\left(\mathbf{r}, \mathbf{r}^{\prime}, \omega\right)=\frac{i}{2 \pi} \iint d \mathbf{k} \mathcal{F}\left(\mathbf{r}, \mathbf{r}^{\prime}, \omega\right) \mathcal{Q}(\mathbf{k}, \omega)
$$

where $\mathcal{F}\left(\mathbf{r}, \mathbf{r}^{\prime}, \omega\right)$ is a spatial function that connects two points $\mathbf{r}$ and $\mathbf{r}^{\prime}$ above the surface:

$$
\mathcal{F}\left(\mathbf{r}, \mathbf{r}^{\prime}, \omega\right)=\exp \left[i \mathbf{k} \cdot\left(\mathbf{l}-\mathbf{l}^{\prime}\right)+i w_{0}\left(z+z_{0}\right)\right]
$$

with $\mathbf{r}=(\mathbf{l}, z), \mathbf{r}^{\prime}=\left(\mathbf{l}^{\prime}, z^{\prime}\right), \mathbf{k}=\left(k_{x}, k_{y}\right)$, and $w_{0}^{2}=k_{0}^{2}-$ $k^{2}$ [with $\left.\operatorname{Im}\left(w_{0}\right) \geqslant 0\right]$. The factor $\mathcal{Q}(\mathbf{k}, \omega)$ is a second rank tensor directly related to the optical response properties of the surface: 


$$
\mathcal{Q}(\mathbf{k}, \omega)=\left(\begin{array}{ccc}
\frac{-k_{x} k_{y} k_{0}}{k^{2}}\left\{\Delta_{p}+\Delta_{s}\right\} & \frac{-k_{0}}{k^{2}}\left\{\Delta_{p} k_{y}^{2}-\Delta_{s} k_{x}^{2}\right\} & \frac{-k_{y} k_{0}}{w_{0}} \Delta_{p} \\
\frac{k_{0}}{k^{2}}\left\{\Delta_{p} k_{x}^{2}-\Delta_{s} k_{y}^{2}\right\} & \frac{k_{x} k_{y} k_{0}}{k^{2}}\left\{\Delta_{p}+\Delta_{s}\right\} & \frac{k_{x} k_{0}}{w_{0}} \Delta_{p} \\
\frac{k_{y} k_{0}}{w_{0}} \Delta_{s} & \frac{-k_{x} k_{0}}{w_{0}} \Delta_{s} & 0
\end{array}\right) .
$$

In this equation, the two refection coefficients $\Delta_{p}$ and $\Delta_{s}$ are functions of the optical dielectric constant $\epsilon_{s}(\omega)$ of the surface

$$
\Delta_{p}=\frac{w-\epsilon_{s}(\omega) w_{0}}{w+\epsilon_{s}(\omega) w_{0}}
$$

and

$$
\Delta_{s}=\frac{w-w_{0}}{w+w_{0}},
$$

with

$$
w=\left[\epsilon_{s} k_{0}^{2}-k^{2}\right]^{1 / 2} .
$$

Note that the retarded character of the information is implicitly contained in the two factors $w$ and $w_{0}$, via the wave vector $k_{0}$. When such effects are assumed to play a minor role, i.e., for example, in the electrostatic approximation, then $\mathcal{Q}(\mathbf{k}, \omega)$ vanishes, as expected.

${ }^{1}$ D. W. Pohl, in Near-Field Optics, Vol. 242 of NATO Advanced Studies Institute Series E: Applied Sciences, edited by D. W. Pohl and D. Courjon (Kluwer, Dordrecht, 1993), pp. 1-5.

${ }^{2}$ D. Courjon and C. Bainier, Rep. Prog. Phys. 57, 989 (1994).

${ }^{3}$ Photons and Local Probes, Vol. 300 of NATO Advanced Studies Institute Series E: Applied Sciences, edited by O. Marti and R. Möller (Kluwer, Dordrecht, 1995).

${ }^{4}$ D. van Labeke and D. Barchiesi, in Near-Field Optics, Vol. 242 of NATO Advanced Studies Institute Series E: Applied Sciences (Ref. 1), pp. 157-178.

${ }^{5}$ A. G. Choo et al., Appl. Phys. Lett. 65, 947 (1994).

${ }^{6}$ E. Bourillot et al., J. Opt. Soc. Am. A 12, 95 (1995).

${ }^{7}$ P. Dawson, F. de Fornel, and J. P. Goudonnet, Phys. Rev. Lett. 72, 2927 (1994).

${ }^{8}$ J. R. Krenn et al., Appl. Phys. A 61, 541 (1995).

${ }^{9}$ D. Courjon, C. Bainier, and F. Baida, Opt. Commun. 110, 7 (1994).

${ }^{10}$ S. I. Bozhevolnyi and B. Vohnsen, Phys. Rev. Lett. 77, 3351 (1996).

${ }^{11}$ C. Girard and D. Courjon, Phys. Rev. B 42, 9340 (1990).

${ }^{12}$ O. J. F. Martin, C. Girard, and A. Dereux, J. Opt. Soc. Am. A 13, 1801 (1996).

${ }^{13}$ R. Carminati and J. J. Greffet, J. Opt. Soc. Am. A 12, 2716 (1995).

${ }^{14}$ H. Furukawa and S. Kawata, Opt. Commun. 132, 170 (1996).

${ }^{15}$ O. J. F. Martin, C. Girard, and A. Dereux, Phys. Rev. Lett. 74, 526 (1995).

${ }^{16}$ C. Girard, A. Dereux, O. J. F. Martin, and M. Devel, Phys. Rev. B 52, 2889 (1995).

${ }^{17}$ D. Barchiesi et al., Phys. Rev. E 54, 4285 (1996).

${ }^{18}$ J. C. Weeber et al., Phys. Rev. Lett. 77, 5332 (1996).

${ }^{19}$ D. Courjon et al., in Photon and Local Probes, Vol. 300 of NATO Advanced Studies Institute Series E: Applied Sciences (Ref. 3), pp. 59-70.

${ }^{20}$ J. C. Weeber, Ph.D. thesis, University of Burgundy, Dijon, 1996.
${ }^{21}$ D. Barchiesi and D. V. Labeke, in Near-Field Optics, Vol. E-242 of NATO Advanced Studies Institute Series E: Applied Sciences, edited by D. Courjon and D. W. Pohl (Kluwer, Dordrecht, 1995), pp. 105-117.

${ }^{22}$ D. Courjon, F. Baida, C. Bainier, and D. van Labeke, in Optics at the Nanometer Scale, Vol. E-319 of NATO Advanced Studies Institute Series E: Applied Sciences, edited by M. NietoVesperinas and N. Garcia (Kluwer, Dordrecht, 1996), pp. 179188.

${ }^{23}$ C. Girard and A. Dereux, Rep. Prog. Phys. 59, 657 (1996).

${ }^{24} \mathrm{R}$. Petit et al., in Electromagnetic Theory of Gratings, edited by R. Petit, Topic in Current Physics Vol. 22 (Springer-Verlag, Heidelberg, 1980).

${ }^{25}$ D. Maystre and M. Nevière, J. Opt. 9, 301 (1978).

${ }^{26}$ F. Montiel and M. Nevière, J. Opt. Soc. Am. A 11, 3241 (1994).

${ }^{27}$ M. Abe and M. Koshiba, J. Opt. Soc. Am. A 11, 2038 (1994).

${ }^{28}$ J. P. Goudonnet et al., J. Opt. Soc. Am. A 12, 1749 (1995).

${ }^{29}$ G. S. Agarwal, Phys. Rev. A 11, 230 (1975).

${ }^{30}$ O. Keller, Phys. Rep. 268, 85 (1996).

${ }^{31}$ A. Dereux, J. P. Vigneron, P. Lambin, and A. A. Lucas, Physica B 175, 65 (1991).

${ }^{32}$ A. Yaghjian, Proc. IEEE 68, 248 (1980).

${ }^{33}$ R. C. Reddick, R. J. Warmack, and T. L. Ferrell, Phys. Rev. B 39, 767 (1989).

${ }^{34}$ D. Courjon, K. Sarayedine, and M. Spajer, Opt. Commun. 71, 23 (1989).

${ }^{35}$ T. L. Ferrell, S. L. Sharp, and R. J. Warmack, Ultramicroscopy 42-44, 408 (1991).

${ }^{36}$ D. Courjon, C. Bainier, and M. Spajer, J. Vac. Sci. Technol. 10, 2436 (1992).

${ }^{37}$ F. de Fornel et al., Ultramicroscopy 42-44, 416 (1992).

${ }^{38}$ N. F. V. Hulst, F. B. Segerink, F. Achten, and B. Bolger, Ultramicroscopy 42-44, 416 (1992).

${ }^{39}$ N. F. V. Hulst, M. H. P. Moers, and B. Bögler, J. Microsc. 171, 95 (1993). 